\title{
Diodos emissores de luz como fonte de bombeio em fotorefletância
}

\section{Light emitting diode as a photoreflectance pump source}

\author{
Leonardo Dias de Souza ${ }^{1}$; Ivan Frederico Lupiano Dias²; Edson Laureto²; \\ Dari Oliveira Toginho Filho²; José Leonil Duarte ${ }^{2}$
}

\section{Resumo}

Diodos emissores de luz (LEDs) com emissão em diferentes comprimentos de onda são empregados como fonte de bombeamento em fotorefletância - PR ("photoreflectance"). São analisados espectros de fotorefletância de semicondutores volumétricos de GaAs e de heteroestruturas GaAs/InGaP e GaAsSbN/ GaAs, preparadas sobre substrato de GaAs, usando LEDs azul, verde, laranja e vermelho, em comparação com espectros obtidos com o emprego de lasers de Ar e He-Ne, em montagem convencional. Nossos resultados demonstram a possibilidade do emprego de LEDs como fonte de bombeamento em medidas de fotorefletância.

Palavras-chave: Fotorefletância. Fonte de bombeamento. Diodos emissores de luz.

\begin{abstract}
Light-emitting diodes (LEDs) with different wavelengths are used as photoreflectance light pump sources. Photoreflectance spectra of bulk semiconductors of GaAs and GaAs/InGaP and GaAsSbN/ GaAs heterostructures prepared on GaAs substrates were investigated, using blue, green, orange and red LEDs, in comparison with spectra obtained with the use of Ar and He-Ne lasers, using a conventional technique. Results show the possibility of using LEDs as pump light sources in photoreflectance measurements.
\end{abstract}

Key words: Photoreflectance. Pump Source. Light-emitting diodes.

\footnotetext{
1 Mestrando em Física, Universidade Estadual de Londrina, C. P. 6001, CEP: 86051-990, Londrina-PR, Brasil. E-mail: leo-xx-londrina@ig.com.br

2 Departamento de Física, Universidade Estadual de Londrina, C. P. 6001, CEP: 86051-990, Londrina-PR, Brasil.
} 


\section{Introdução}

A fotorefletância (PR) é uma técnica experimental não destrutiva, que permite a eletromodulação sem contato, para o estudo e a caracterização de várias propriedades ópticas de materiais e estruturas de materiais semicondutores (POLLAK, 1993). Essa técnica foi concebida ainda na fase inicial do desenvolvimento da espectroscopia por modulação, na década de 60, mas devido a dificuldades experimentais para análise do sinal permaneceu relativamente esquecida até meados da década de 80 , quando foi revivida pelos trabalhos de Glembocki et al. (1985 a e b).

A técnica de PR vem-se mostrando ser uma ferramenta experimental poderosa para o estudo de propriedades ópticas de diversos materiais semicondutores volumétricos ("bulk") (CHANG, 2005), de baixa dimensionalidade (KUDRAWIEC et al., 2006), (RUDNO-RUDZINSKY et al., 2005), (RIBEIRO et al., 2000), em dispositivos (MURTAGH et al., 2004) e para análise "in situ” em técnicas de crescimento epitaxial, como epitaxia por feixe molecular- MBE ("molecular beam epitaxy") (ZHOU et al., 1993).

Por outro lado, os sistemas convencionais de PR apresentam algumas dificuldades. São elas relacionadas aoemprego de seccionadores mecânicos de luz ("choppers"), que impõem restrições para a freqüência de operação, e apresentam problemas na estabilidade para rotação a altas freqüências; à ocorrência de sinais espúrios no fotodetetor devido ao espalhamento da luz de bombeio pela amostra e/ou fotoluminescência a baixas temperaturas; e às dificuldades nas análises do sinal devido à superposição de sinais de várias camadas e interfaces existentes nas amostras, e/ou forte absorção de luz nas camadas mais internas, devido ao emprego de fontes de bombeio com comprimento de onda fixo.

Vários métodos e alternativas técnicas têm sido desenvolvidos para superação desses problemas, como o emprego de "choppers" acusto-ópticos
(SHEN et al., 1990); servo-normalização com o uso de filtros de densidade neutra variáveis - VNDF (“variable neutral density filter")(SHEN et al., 1987); fotorefletância diferencial - DPR ("differential PR") (SYDOR et al., 1991); "dye” laser sintonizável como feixe de prova (GLEMBOCKI; SHANABROOK, 1987); monocromadores duplos (THEIS et al., 1988); fontes duplas de bombeamento com comprimento de onda fixos (TANG, 1992); bombeamento com luz branca e dois "choppers" de luz - DCPR ("dual chopped PR") (LU et al., 1990), (CHANDLERHOROWITZ, 1995), (GHOSH; ARORA, 1995); e LEDs azuis de GaN como fontes de bombeamento (GASKILL; HOLM; GLEMBOCKI, 2000).

A opção original pelo emprego de LEDs como fonte de bombeio apresentada por Gaskill e colaboradores (GASKILL; HOLM; GLEMBOCKI, 2000), parece bastante interessante e eficaz, pois possibilita a superação de vários dos problemas apresentados e também abre novas possibilidades técnicas. Nessa alternativa técnica, a fonte de bombeamento, geralmente lasers ou luz branca, é substituída por LEDs e o “chopper” é substituído por um gerador de freqüências permitindo a modulação eletro-ótica do sinal. Isso permite atacar alguns dos problemas técnicos citados e tem a vantagem de permitir o emprego de fontes de excitação em um grande intervalo de comprimento de onda, dada a disponibilidade e o baixo custo de LEDs no mercado. Gaskill e colaboradores (GASKILL; HOLM; GLEMBOCKI, 2000) empregaram três LEDs de $\mathrm{GaN}$, com emissão em 422, 455 e 525 nm, para medidas de impedância óptica com fotorefletância em amostras de GaAs $\mathrm{SIN}^{+}$, orientados (001), com camada "cap" de $\mathrm{Fe}^{20}$. A aplicação desta técnica ocorreu em um tipo específico de amostra, e em um intervalo relativamente estreito de comprimento de onda. Além disso, não foram disponibilizados no texto citado espectros de PR que permitissem uma análise comparativa entre as medidas realizadas com lasers e LEDs. No melhor de nosso conhecimento, não temos informação de trabalhos recentes empregando a mesma técnica. 
Neste trabalho, analisamos o comportamento da fotorefletância em material semicondutor volumétrico (GaAs), e em duas heteroestruturas de poços quânticos, um não-tensionado ( $\mathrm{GaAs} / \mathrm{InGaP})$ e outro tensionado (GaAsSbN/GaAs). Ambos foram preparados sobre substrato de GaAs, e empregaramse LEDs em um grande intervalo de comprimentos de onda - azul, verde, laranja e vermelho, em comparação com fontes de bombeio tradicionais como lasers de He-Ne e Ar. Verifica-se que a fotorefletância com LEDs pode ser uma alternativa adequada para a caracterização de propriedades ópticas de materiais e heteroestruturas de materiais semicondutores.

\section{Detalhes experimentais}

Empregaram-se quatro LEDs de diferentes materiais, InGaN (azul), GaP/GaP (verde) e AlGaInP (laranja e vermelho), fabricados pela Optech [http:// www.optech.ind.br/], com energias de emissão (meia largura a meia altura FWHM - "Full Width Half Maximum") de respectivamente 2,701 $(0,191)$, 2,400 (0,171), 1,984 (0,053) e 1,868 eV (0,072 eV).

Os LEDs foram montados em uma cápsula metálica cilíndrica aterrada para minimizar a detecção da radiação eletromagnética emitida pelo LED no fotodetetor da montagem de PR, reproduzindo procedimento mencionado por Gaskill e colaboradores (GASKILL; HOLM; GLEMBOCKI, 2000). O gerador de freqüência empregado para alimentar o LED é um gerador modelo MFG4202 fabricado pela Minipa [http://www.minipa. com.br/]. Foi utilizada uma alimentação na forma de onda quadrada com freqüência de $200 \mathrm{~Hz}$. As medidas empregando LEDs foram comparadas com medidas extraídas com uma montagem utilizando lasers de He-Ne com emissão em 1,962 eV e de $\mathrm{Ar}^{+}$sintonizado em 2,41 eV (verde) e em 2,708 eV (azul), ambos com intensidade luminosa de $1 \mathrm{~mW} /$ $\mathrm{cm}^{2}$. A intensidade luminosa dos LEDs sobre as amostras foi estimada em aproximadamente $10 \mathrm{~mW} /$ $\mathrm{cm}^{2}$. Para a detecção do sinal das amostras, foram utilizados um detector de silício (\#1 e \#2) e um detector de InGaAs (\#3). Na figura 1, é apresentada a montagem utilizada nas medidas de PR.

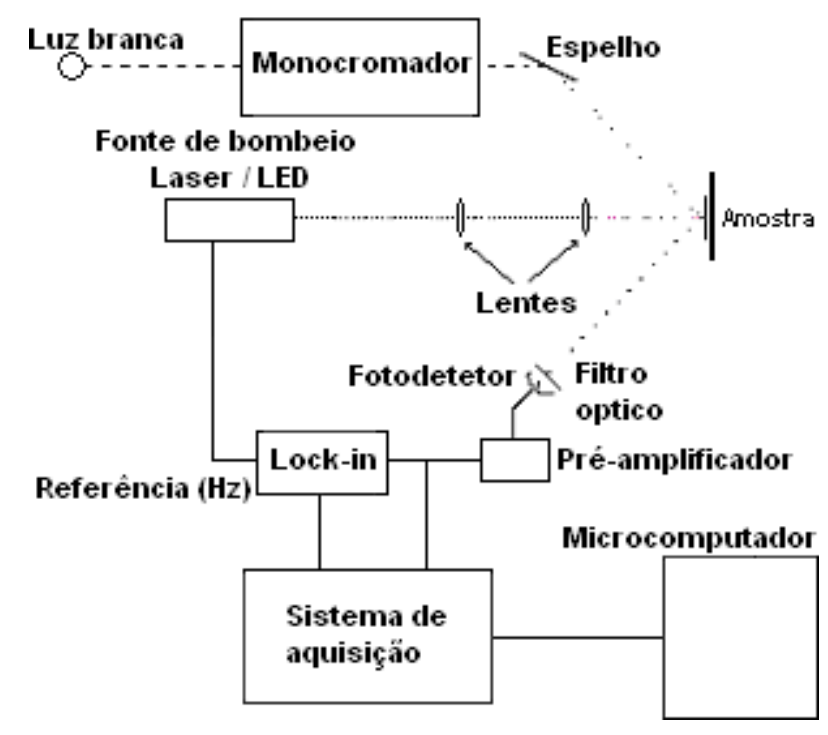

Figura 1. Diagrama da montagem experimental utilizada nas medidas de fotorefletância.

Foram analisadas três amostras que continham GaAs: a amostra \#1 consiste de GaAs volumétrico ("bulk") com $1 \mathrm{~m}$ de espessura; na amostra \#2, o GaAs é a camada ativa de uma estrutura de poço quântico simples não tensionado, constituído de barreiras de $\mathrm{In}_{0.48} \mathrm{Ga}_{0.52} \mathrm{P}$ com espessura de $500 \AA$ e poço de $60 \AA$; e na amostra \#3, o GaAs forma as barreiras de potencial em uma estrutura de poço quântico tensionado, onde a camada ativa é de $\mathrm{GaAs}_{0.843} \mathrm{Sb}_{0.15} \mathrm{~N}_{0.007}$ com $150 \AA$ de espessura. Essa estrutura está inserida entre duas camadas de confinamento ("cladding") de AlGaAs. O crescimento das amostras \#1 e \#3 foi feito por Epitaxia por Feixe Molecular no Laboratório de Semicondutores da USP e no Laboratoire de Photonique et de Nanostructures (NRS, França), enquanto o da amostra \#2 foi feito por Deposição por Vapor Químico de Organometálicos na UNICAMP. Todas as amostras foram preparadas sobre substrato semi-isolante de GaAs. Todas as medidas de PR foram realizadas a temperatura ambiente $(300 \mathrm{~K})$. 


\section{Resultados experimentais e discussão}

Os círculos vazios nas Figuras 2-(a), 2(b) e 2-(c) representam, à temperatura ambiente, os espectros de PR das amostras \#1, \#2 e \#3, respectivamente, e utilizam como feixe do bombeio um laser de $\mathrm{Ar}^{+}$ sintonizado em 2,41 eV. O sinal da amostra \#1 é constituído de uma estrutura em torno de 1,42 $\mathrm{eV}$, relacionada à transição do "gap" fundamental $\left(\mathrm{E}_{0}\right)$ do GaAs, seguida por oscilações rapidamente amortecidas, provavelmente devidas ao efeito Franz-Keldysh (SHEN; DUTTA, 1995). Seguindo a teoria desenvolvida por Aspnes (ASPNES, 1980), a posição em energia do n-ésimo extremo dessas oscilações obedece à relação

$$
n \pi=(4 / 3)\left[\left(E_{n}-E_{g}\right) / \hbar \theta\right]^{3 / 2}+\chi
$$

onde, $E_{n}$ é a energia do n-ésimo extremo, $E_{g}$ é a energia do "gap" fundamental, e $\hbar \theta$ é a energia eletro-óptica, relacionada com a intensidade do campo elétrico interno através de

$$
(\hbar \theta)^{3}=q^{2} \hbar^{2} F^{2} / 2 \mu \|
$$

onde, $q$ é a carga do elétron, $F$ é a intensidade do campo elétrico, e $\mu \|$ é a massa reduzida interbandas na direção do campo.

Na Figura 2-(a) estão indicados em seqüência os extremos das oscilações. O "inset” na Figura 2-(a) é um gráfico do índice dos extremos das oscilações em função de $(4 / 3)\left(E_{n}-E_{g}\right)^{3 / 2}$, representados por círculos preenchidos. Percebe-se que esses pontos são muito bem descritos por uma reta, o que indica tratar-se realmente de oscilações geradas pelo efeito Franz-Keldysh. Foi feito um ajuste linear sobre esses pontos, indicado no "inset" pela linha contínua, e de sua inclinação obtém-se $(\hbar \theta)^{-3 / 2}$. Fazendo uso da
Eq. (2) e utilizando $\mu \|$ igual a $0,057 m_{0}$, onde $m_{0}$ é a massa em repouso do elétron, foi encontrado um valor de $10.6 \mathrm{kV} / \mathrm{cm}$ para a intensidade $F$ do campo elétrico interno nesta amostra.

$\mathrm{Na}$ seqüência, são analisados os espectros de PR das amostras \#2 e \#3. As linhas contínuas nas Figs. 2-(b) e 2-(c) são ajustes realizados sobre os pontos experimentais utilizando a forma de linha tipo terceira derivada proposta por Aspnes (ASPNES, 1980):

$$
\Delta R / R=\operatorname{Re}\left[A e^{i \phi}\left(E-E_{j}+i \Gamma\right)^{-m}\right] .
$$

Nesta relação, $A$ é a amplitude, $\phi$ a fase, $E_{j}$ a energia, e $\Gamma$ a largura de linha da transição, enquanto $m$ é um parâmetro que depende da dimensionalidade do ponto crítico. Nos ajustes mostrados nas Figuras 2-(b) e 2-(c), utilizou-se $m=3$ para as transições que envolviam níveis confinados (GEDDO et al., 2003).

O espectro da amostra \#2 (Fig. 2-(b)) é composto de estruturas provenientes de várias transições, e as três de menor energia estão centradas em 1,421 $\mathrm{eV}, 1,479 \mathrm{eV}$ e 1,510 eV, e são relacionadas com a transição $\mathrm{E}_{0}$ da camada buffer do GaAs e com as transições envolvendo o primeiro nível de energia do elétron e os primeiros níveis de buraco pesado (e1-hh1) e leve (e1-lh1) no poço quântico, respectivamente. Estas atribuições são baseadas em estimativas teóricas da energia destas transições, utilizando o modelo de massa efetiva. As demais estruturas do espectro são provenientes de transições envolvendo estados excitados do poço. Já o espectro da amostra \#3 (Fig. 2-(c)) é ajustado com quatro estruturas, centradas em 1,055 eV, 1,095 eV, 1,135 $\mathrm{eV}$ e $1,235 \mathrm{eV}$, respectivamente, todas relacionadas com transições ópticas envolvendo os diferentes níveis de energia do poço quântico tensionado. 


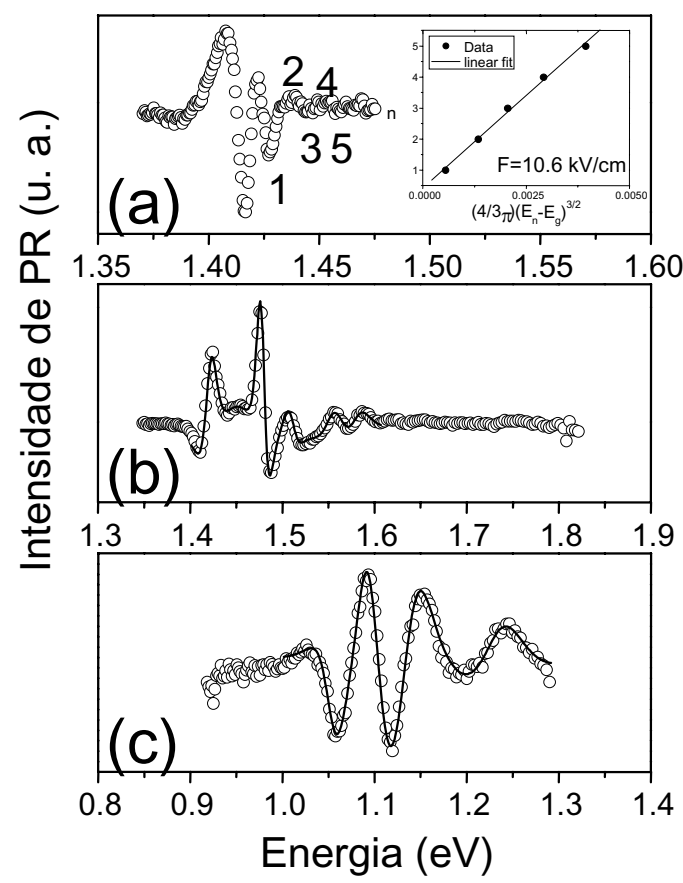

Figura 2. Espectros de PR a temperatura ambiente (círculos vazios) da amostra \# 1 (a), \# 2 (b) e \# 3 (c). $\mathrm{O}$ "inset" em (a) é o gráfico do índice n do extremo da oscilação contra $(4 / 3 \pi)\left(E_{n}-E_{g}\right)^{3 / 2}$ (veja-se texto para pormenores). As linhas contínuas em (b) e (c) são curvas de ajuste usando a eq. (3).

Nas Figuras 3, 4 e 5 estão mostrados os espectros das amostras \#1, \#2 e \#3, respectivamente, obtidos fazendo-se uso das diferentes fontes de bombeio de lasers e LEDs. Pode-se observar que a forma dos espectros é praticamente insensível ao feixe de bombeamento empregado, e as diferentes estruturas que compõem os espectros têm sua forma de linha basicamente mantida, independentemente do laser ou do LED utilizado durante a medida. A menor relação sinal/ruído em algumas medidas indica ser necessário ainda uma otimização das condições experimentais.

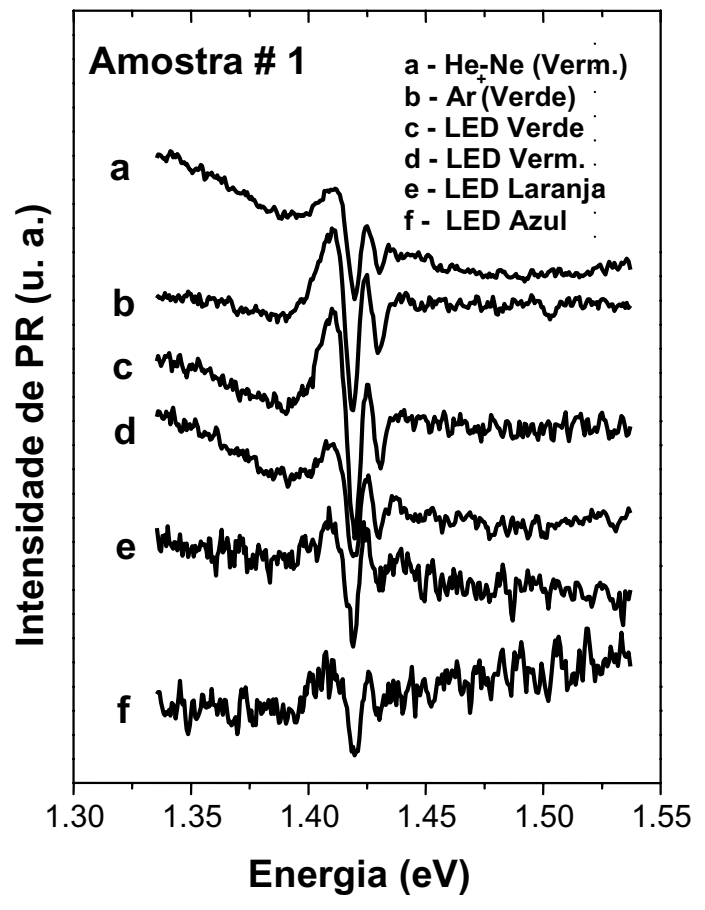

Figura 3. Espectro de PR para a amostra \# 1, usando diferentes fontes de bombeio (olhar legenda para correlacionar a curva com a fonte de bombeio).

Pequenas variações na intensidade de emissão dos LEDs, associadas provavelmente ao circuito de modulação devido às diferentes correntes de operação para cada LED, podem estar influenciando a magnitude da relação sinal/ruído. Em geral, o nível de ruído aumenta quando variamos a energia do feixe de bombeio no sentido do azul. Isso também está associado a uma maior dispersão dos elétrons fotogerados na amostra, devido ao excedente de energia proporcionado pela grande energia de excitação em relação às energias das transições sob análise. Os espectros de PR na amostra de $\mathrm{GaInP} / \mathrm{GaAs}$, entretanto, não mostram variação significativa do padrão sinal/ruído para todas as fontes de bombeio. Estes resultados demonstram a eficácia dos LEDs na modulação óptica de PR. 


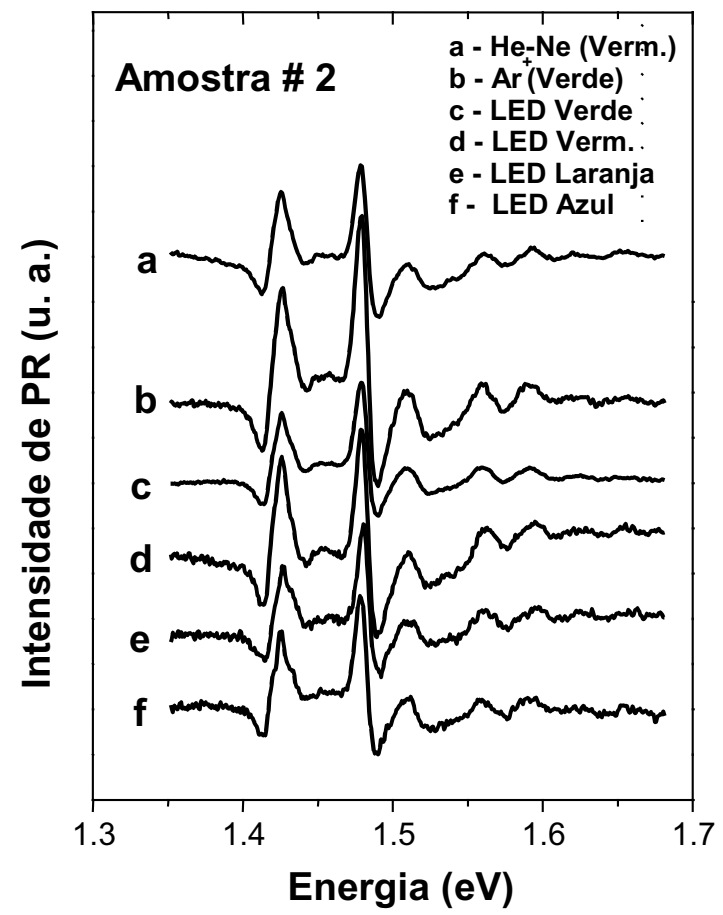

Figura 4. Espectro de PR para a amostra \# 2, usando diferentes fontes de bombeio (olhar legenda para correlacionar a curva com a fonte de bombeio).

Parece existir um consenso nos artigos científicos de que o sinal de PR vem da modulação do campo elétrico interno, existente em alguma interface ou na superfície (interface semicondutor/ar) da amostra (POLLAK; SHEN, 1993), (SHEN; DUTTA, 1995), (CHOUAIB et al., 2005), por meio da injeção de pares elétron-buraco fotogerados. Sugere-se também que o mecanismo de modulação que dá origem ao sinal de PR em poços quânticos está relacionado com as variações periódicas induzidas pelo feixe de bombeio na população dos níveis do poço, o que afeta o perfil de potencial e, por sua vez, modifica os estados de energia (efeito Stark quântico confinado) (TANG, 1991).

Uma investigação do mecanismo de modulação responsável pelo sinal de PR de uma determinada heteroestrutura poderia ser feita mediante a "sintonização" da energia dos fótons do feixe de bombeamento, gerando portadores acima ou abaixo do "gap" dos diferentes materiais que compõem a heteroestrutura. Isso pode ser facilmente realizado, com baixo custo, utilizando-se um conjunto de LEDs com diferentes energias de emissão.

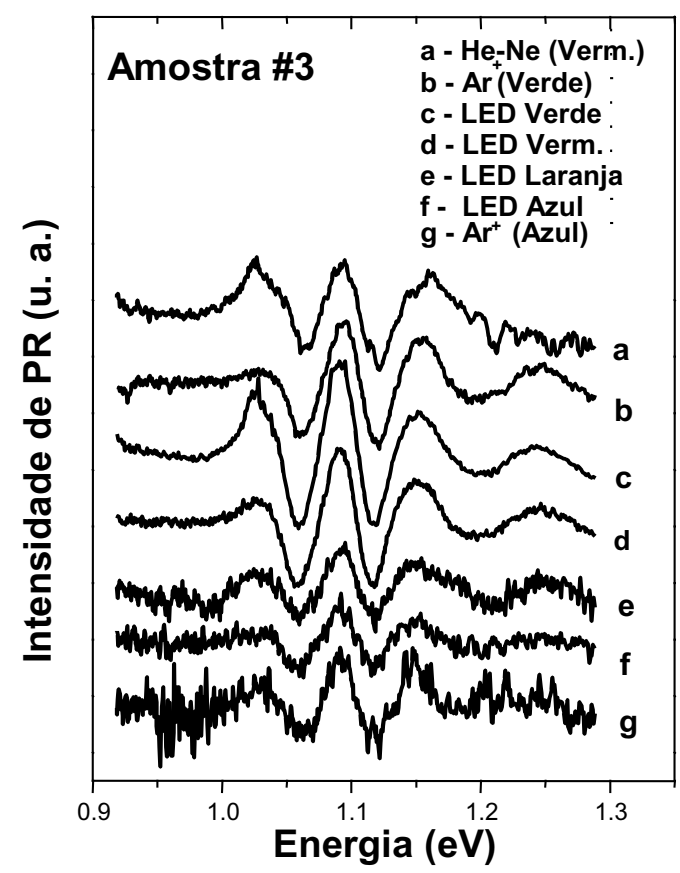

Figura 5. Espectro de PR para a amostra \# 3, usando diferentes fontes de bombeio (olhar legenda para correlacionar a curva com a fonte de bombeio).

Outra possibilidade é utilizar o amplo intervalo de freqüências admitido pelo gerador de ondas para estudos da amplitude do sinal de PR, em função da freqüência da modulação, como mostrado por Gaskill, Holm e Glembocki (2000).

\section{Conclusão}

Em conclusão, neste trabalho foi demonstrado e avaliado o emprego de LEDs como fonte de bombeio em experimentos de PR, em um amplo intervalo de comprimento de onda do feixe. Verificou-se que a energia da fonte de bombeamento não interfere significativamente na forma de linha do espectro de PR. A modulação foi feita eletronicamente, utilizando um gerador de ondas como fonte de alimentação para o LED, eliminando-se a necessidade de utilizar um "chopper" para fornecer a referência ao amplificador "lock-in". Assim, o 
emprego de LEDs diminui consideravelmente o custo de implementação da técnica de PR, além de abrir novas perspectivas de investigação, com a sintonização da energia dos fótons, levando em conta os "gaps" característicos da amostra estudada, ou em função da freqüência e formato da onda da fonte de alimentação do LED.

\section{Agradecimentos}

Os autores agradecem o suporte financeiro dado pelas agências CAPES, CNPq, Fundação Araucária e Fundação Banco do Brasil.

\section{Referências}

ASPNES, D. E. Modulation spectroscopy/electric field effects on the dieletric function of semiconductors. In: MOSS, T. S., BALKANSKI, M. (Ed.). Handbook on semiconductors. Amsterdam: North-Holland, 1980. v. 2, Cap. 4A. p. 109.

CHANDLER-HOROWITZ, D.; BERNING, D. W.; PELlEGRINO, J. G.; BURNETT, J. H.; AMRITHARAJ, P. M.; BOUR, D. P.; TREAT, W. D. In: BULLINS, W. M., SEILLER, D. G., DIEBOLD, A. C. (Ed.). Proceedings of the International Workshop Semiconductor Characterization. New York: Institute Physics Conference Series. 1995. p. 639-641.

CHANG, C. H.; WANG, D. P.; WU, C. C.; HSIAO, C. L.; TU, L. W. Contactless eletroreflectance and photoreflectance studies of n- and p-type-doped GaN with Ga and N face. Applied Physics Letters, New York, v. $87,202103,2005$.

CHOUAIB, H.; BRU-CHEVALLIER, C.; GUILLOT, G.; LAHRECHE, H.; BOVE, P. Photoreflectance study of $\mathrm{GaAsSb} / \mathrm{InP}$ heterostructures. Journal of Applied Physics, New York, v. 98, n. 12, p. 1-7, 2005.

GASKILL, D. R.; HOLM, R. T.; GLEMBOCKI, O. J. GaN light emitting diode as a photoreflectance pump source. Review Scientific Instruments, New York, v. 71, n. 11, p. 4341-4344, 2000.

GEDDO, M.; GUIZETTI, G.; CAPIZZI, M.; POLIMENI, A.; GOLLUB, D.; FORCHEL, A. Photoreflectance evidence of the $\mathrm{N}$-induced increase of the exciton binding energy in an $\operatorname{In}_{\mathrm{x}} \mathrm{Ga}_{1-\mathrm{x}} \mathrm{As}_{1-\mathrm{y}} \mathrm{N}_{\mathrm{y}}$ alloy. Applied Physics Letters, New York, v. 83, n. 3, p. 470-472, 2003.
GHOSH, S.; ARORA, B. M. Double AC photoreflectance spectroscopy of semiconductors. IEEE Journal of Selected Topics in Quantum Electronics, New York, v. 1, n. 4, p. 1108-1112, 1995.

GLEMBOCKI, O. J.; SHANABROOK, B. V. Photoreflectance characterization of GaAs/AlGaAs thin-films, heterojunctions and multiple quantum well structures. Proceedings of the Society Photo-Optical Instrumentation Engineering, San Diego, v. 794, p.7477, 1987.

GLEMBOCKI, O. J.; SHANABROOK, B. V.; BOTTKA, N.; BEARD, W. T.; COMAS, J. Photoreflectance characterization of gallium arsenide/aluminum gallium arsenide thin films, heterojunctions and multiple quantum well structures. Proceedings of the Society Photo-Optical Instrumentation Engineering, San Diego, v. 524, p. 8689, 1985a.

GLEMBOCKI, O. J.; SHANABROOK, B. V.; BOTTKA, N.; BEARD, W. T.; COMAS, J. Photoreflectance characterization of interband transitions in $\mathrm{GaAs} /$ AlGaAs multiple quantum wells and modulation-doped heterojunctions. Applied Physics Letters, New York, v. 46, n. 10, p. 970-972, 1985b.

KUDRAWIEC, R.; GLADYSIWIECZ, M.; MISIEWICZ, J.; YUEN, H. B.; BANK, S. R.; WISTEY, M. A.; BAL, H. P.; HARRIS, J. S. Photoreflectance spectroscopy of a $\mathrm{Ga}_{0.62} \mathrm{In}_{0.38} \mathrm{~N}_{0.026} \mathrm{As}_{0.954} \mathrm{Sb}_{0.02} / \mathrm{GaAs}$ single quantum well tailored at $1.5 \mu \mathrm{m}$. Solid State Communications, New York, v. 137, n. 3, p.138-141, 2006.

LU, C. R.; ANDERSON, J. R.; STONE, D. R.; BEARD, W. T.; WILSON, R. A. Photoreflectance study of the internal electric fields at the n-type GaAs surface and across the n-type GaAs/substrate interface. Superlattices Microstructures, Amsterdam, v. 8, n. 2, p.155-157, 1990.

MURTAGH, M. E.; GUENEBAUT, V.; WARD, S.; NEE, D.; KELLY, P. V.; LOONEY, B. O.; MURPHY, F.; MORDREANU, M.; WESTWATER, S.; BLUST, R.; BLAND, S. W. Photoreflectance spectroscopy study of vertical cavity surface emitting laser structures. Thin Solid Films, Lausanne, v. 450, n. 1, p.148-150, 2004.

POLLAK, F. H.; SHEN, H. Modulation spectroscopy of semiconductors: bulk/thin film, microstructures, surfaces/interfaces and devices. Materials Science and Engineering, Lausanne, v. 10, n.7-8, p. 275-374, 1993.

RIBEIRO, E.; CERDEIRA, F.; BRASIL, M. J. S. P.; HEIZEL, T.; ENSSLIN, K.; MEDEIROS-RIBEIRO, G.; PETROFF, P. M. An optical study of self-assembled 
In $\mathrm{Ga}_{1-\mathrm{x}} \mathrm{As} / \mathrm{GaAs}$ quantum dots embedded in a twodimensional electron gas. Journal of Applied Physics, New York, v. 87, n. 11, p. 7994-7998, 2000.

RUDNO-RUDZINSKY, W.; RYCZKO, K.; SEK, G.; MISIEWICZ, J.; DA SILVA, M. J.; QUIVY, A. A.. Photoreflectance study of energy level structure of selfassembled InAs/GaAs quantum dots emitting at $1.3 \mathrm{~m}$. Solid State Communications, New York, v. 135, n. 4, p. 232-236, 2005.

SHEN, H.; DUTTA, M. Franz-Keldysh oscillations in modulation spectroscopy. Journal of Applied Physics, New York,v. 78, n. 4, p. 2151-2153, 1995.

SHEN, H.; POLLAK, F. H.; WOODALL, J. M.; SACKS, R. N. Photoreflectance study of electric field distributions in semiconductor heterostructures grown on semiinsulating substrates. Journal of Electronic Materials, Warrendale, v. 19, n. 3, p. 283-287, 1990.

SHEN, H.; PARAYANTHAL, P.; LIU, Y. F.; POLLAK, F. H. New normalization procedure for modulation spectroscopy. Review Scientific Instruments, New York, v. 58, n. 8 , p. $1429-1432,1987$.
SYDOR, M.; BADAKLSHAN, A.; ENGHOLM, J. R.; DALE, D. A. Differential photoreflectance from modulation-doped heterojunctions. Applied Physics Letters, New York, v. 58, n. 9, p. 948-950, 1991.

TANG, Y.S.Photoreflectanceline shapes of semiconductor microstructures. Journal of Applied Physics, New York, v. 69 , n. 12 , p. 8298-8303, 1991.

TANG, Y. S. Understanding photoreflectance of modulation-doped heterojunctions. Physica Status Solidi A, Berlin, v. 129, n. 1, p.285-290, 1992.

THEIS, W. M.; SANDERS, G. D.; LEAK, C. E.; BAJAJ, K. K.; MORKOÇ, H. Excitonic transitions in GaAs/ GaxAl1-xAs quantum wells observed by photoreflectance spectroscopy: Comparison with a first-principles theory. Physical Review B, New York, v. 37, n. 10, p. 3042-3051, 1988.

ZHOU, W.; DUTTA, M.; SHEN, H.; PAMULAPATI, J.; BENETT, B. R.; PERRY, C. H.; WEYBUENE, D. $\mathrm{W}$. Investigation of near interface properties in semiinsulating InP substrates with epitaxial grown InGaAs and InAlAs by photoreflectance. Journal of Applied Physics, New York, v. 73, n. 3, p. 1266-1271, 1993. 